\title{
Application of the Transformer Fault Diagnosis Expert System based on Improved Bayesian Rule
}

\author{
Qiming Cui ${ }^{1}$, Kai Liang \\ Liaoning Anshan Power Supply Company, Anshan, 114001, China \\ E-mail: ospb2002@sina.com
}

\section{You Zhou}

Xian Jiaotong University, Xi'an, 710049, China

\section{Shuting Cui}

Eastern Michigan University, Ypsilanti, MI 48197, U.S.A.

\section{Pan Hu}

Southeast University, Nanjing, 211189, China

\section{Yiming Li}

Liaoning Vocational College of Light Industry, Dalian, 116000, China

\section{Nuodi Wang}

Shenyang Institute of Engineering, Shenyang, 110136, China

\section{Xiaoqing Li, Daojun Sun, Yanping Zhang, Dan Wang, Chengwei Han}

Liaoning Anshan Power Supply Company, Anshan, 114001, China

\begin{abstract}
An application research work had improved a Bayesian network rule function of Pro/3 (a production system type expert system shell). This improvement makes it possible for the same rules to ask the same evidence such as the evaluated probability or the certainty factor only once, which can avoid repetitive ask, adding simplicity and improving performance of Pro/3 system. By using it, this work had also created a transformer component fault diagnosis knowledge base model based on the Bayesian network rule so that using the model had implemented an expert system prototype system. The basic application shows the effectiveness of the improvement and the prototype system.
\end{abstract}

CENet2015

12-13 September 2015

Shanghai, China

${ }^{1}$ Speaker 


\section{Introduction}

At present, many researchers have already studied the fault diagnosis of transformer (or power system) by related techniques[1]. Bayesian network with the theoretical basis of strict is a kind of model used to represent (or reasoning) some uncertainty knowledge[2]. An expert system can solve some difficult problems by using some knowledge of experts and reasoning processes[3]. Transformer fault diagnosis generally can identify some fault components by measuring and analyzing some change information in relation to the gas content in transformer oil and electrical index in transformer upon a fault[4,5].

This paper first introduces Bayesian network and rules of the expert system shell Pro/3, and then discusses the function improvement of Bayesian network rules in the Pro/3. On this basis, a transformer component fault diagnosis model based on the part rules of corporate standards of State Grid (Q/GDW 169-2008) « Guide for Condition Evaluation of Oil-immersed Power Transformers(Reactors) » is given with relavant reasoning (computing) and analysis done. The paper also shows the improved part function codes.

\section{Brief Introduction to Bayesian Network and Rules in the Pro/3}

\subsection{Bayesian Network and Diagnosis Inference of the Bayesian Network}

Bayesian network is a causal probabilistic network. It is essentially a concise representation of the joint probability distribution of between some variables. As to a set of variables such as $P\left(x_{1}, x_{2}, \ldots, x_{n}\right)$, then the joint probability density may be describ-ed as follows:

$$
P\left(x_{1}, x_{2}, \ldots, x_{n}\right)=P\left(x_{n} \mid x_{n-1, \ldots,} x_{1}\right) P\left(x_{n-1} \mid x_{n-2, \ldots,} x_{1}\right) \ldots P\left(x_{2} \mid x_{1}\right) P\left(x_{1}\right)
$$

Namely, the joint probability distribution can be broken down into conditional probability. In the Bayesian network, calculation of a child node conditional probability from a parent node is called as the causal reasoning. On the contrary, calculation of a parent node conditional probability from a child node, namely inferencing some causes from some results, is called as the diagnosis reasoning. Some prior probabilities of conditional probability can be obtained from a large amount of historical data through statistical analysis, but also is given (or hypothesised) from some knowledges or experience of the field experts.

\subsection{Bayesian Network Rules in the Pro/3}

Pro/3 includes some inexact reasoning rules with certainty factors and probabilities such as Bayesian network rule and fuzzy sets[6]. In the Pro/3, the call result of Bayesian network rules are considered as two kinds of values: probability and certainty factors. A Bayesian network rules return the certainty factor (or probability) by applying assumptions (built in to the rule) of dependent probabilities on the certainty factors (or probabilities) returned by one or more called rules (which return probabilities (or certainty factors))[7].

\subsection{Increment and Decrement Factor of the Bayesian Network Rules in the Pro/3}

As to each so-called rule, Bayesian network specifies an increment factor (INC) and a decrement factor (DEC). These factors define the significance of the dependency between the propositions represented by the called and the calling rule, namely, the degree of increase in belief (or disbelief) in a proposition $d$ when another proposition $\mathrm{s}$ is specified by a INC (and DEC) [7]. The application of the factors depend on the prior probability (PP, specified when a certainty rule is defined or stated prior to the rule evaluation) and evaluated probability ( $\mathrm{P}$, a post-evidence probability given by a user during expert system running) of the called rule: when $\mathrm{P}>\mathrm{PP}$, the INC has effect; when $\mathrm{P}<\mathrm{PP}$, the DEC has effect; when $\mathrm{P}=\mathrm{PP}$, the factors shall have no effect. The increment and decrement factors are either explicitly specified in the Bayesian rules, or they are specified indirectly by a call to another rule [7].Bayesian network rule evaluation 
always begins from a root rule, but its subrules must be firstly evaluated before evaluation of each rule.

\section{Improvement of the Bayesian Network Rule Function in the Pro/3}

When some Bayesian network rules are evaluated in the original Pro/3, all same rules (or propositions) need (many times) to provide the same evidence (probability or certainty factor). Namely, the Pro/3 does not record the same rule (or proposition) evidence that has ever provided when Bayesian network rules of the original Pro/3 are evaluated. The same rule (or proposition) evidence provided repeatedly (open a same question window) not only affects the efficiency of reasoning but also loses the system simplicity. See Fig.1, the $C_{1}$ and $C_{2}$ correspond to the causes of fault, the $\mathrm{X}_{1}, \mathrm{X}_{2}, \mathrm{X}_{3}, \mathrm{X}_{4}$ and $\mathrm{X}_{5}$ correspond to the symptoms of fault. $\mathrm{C}_{1}$ and $\mathrm{C}_{2}$ have the same rule evidence corresponding to $X_{1}, X_{2}$.

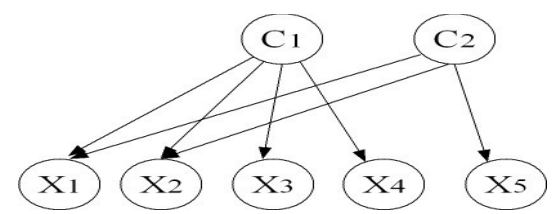

Figure 1: Part Example of the Bayesian Network Rules in the Application Research

\subsection{Basic Idea of the Improvement}

When evaluating (diagnostic reasoning) a Bayesian network rule such as $\mathrm{C}_{1}$ in Fig.1, the Pro/ 3 asks some evidences (evaluated probability or certainty factor) of rule $X_{1}, X_{2}, X_{3}$ and $X_{4}$, and saves them to some data files. The data files include 1) rulename.txt: recording the rules name (some sentences); 2) rule evidence.txt: recording the rules name and their evidences. When the rule $\mathrm{C}_{2}$ is evaluated, the Pro/3 compares some rules to be asked with the content of rulename.txt. If the same rules such as $\mathrm{X}_{1}$ or $\mathrm{X}_{2}$ exist, Pro/3 will not open a window required to provide evidence to avoid repetition, but reads the rules name (belonging to the rule $\mathrm{C}_{1}$ or other) and their evidences from ruleevidence.txt and then writes them to a prolog list in order to match the same rule name such as $\mathrm{X}_{1}$ or $\mathrm{X}_{2}$, and get the corresponding evidences. The improvement was carried out in ir.pro file and taskWindow.pro. Another improvement is that $\mathrm{sx}$ :: fixFormatR (Prior, 2,0, SPrior) which is changed to sx:: fixFormatR (Prior, 3,0, SPrior) in the inexgui.pro in order to display a correct value in the prior probability field (as Fig.5 shows, before changed, the value is 0.00 ) which belongs to definition of Bayesian and Question rule in the Pro/3. A basic flow of the improvement is shown in Fig. 2.

\section{Application Research Based on the Improved Bayesian Network Rules}

The application research is aiming at transforming the overheating fault diagnosis. The knowledge base in respect of fault diagnosis rules can be created by Literature [8]. Part of the rules (described by some English sentences) are shown in Table 1. They are some inexact rules in Pro/3. There are two Bayesian network rules called four or three question rules respectively (corresponding to Fig.1). Table 2 shows two group conditional probabilities: $P\left(d_{1} \mid s_{1} S_{2} S_{3} S_{4}\right)$, $P\left(d_{2} \mid s_{1} S_{2} S_{5}\right)$ (computed by the Bayesian network) under seven prior probabilities based on some experience, five assumptions evaluate probabilities and five increment/decrement values (some combinations). $P\left(d_{1} \mid s_{1} S_{2} S_{3} S_{4}\right)$ and $P\left(d_{2} \mid s_{1} S_{2} S_{5}\right)$ give the possibilities of occurrence of $d_{1}$ and $d_{2}$ type fault when the symptom sign $S_{1}, S_{2}, S_{3}, S_{4}$ or $S_{5}$ are observed. Fig. 3 is the result of the diagnostic reasoning of Bayesian network rules. The fourth column data ( $P\left(d_{1} \mid S_{1} S_{2} S_{3} S_{4}\right)$ ,$\left.P\left(d_{2} \mid s_{1} S_{2} S_{5}\right)\right)$ in Table 2 respectively corresponds to the first and the second row data in Fig. 3. Fig. 4 is a sentence rule graph of calling to the Bayesian network rules. Fig. 5 gives the defined graph of the rule "structure or magnetic shield short circuit" $\left(d_{1}\right)$. Diagnostic reasoning 
is based on following conditional probability computing. According to Formula (1) and conditional prob-ability formula such as $P(A \mid B)=P(A B) / P(B), P(A B)=P(B \mid A) P(A)$, we may get $P\left(d \mid s_{1}\right)=P\left(d s_{1}\right) / P\left(s_{1}\right), P\left(d s_{1}\right)=P\left(s_{1} \mid d\right) P(d)$, and

$$
\begin{aligned}
& P\left(s_{1} s_{2} s_{3}\right)=P\left(s_{1} \mid s_{2} s_{3}\right) P\left(s_{2} \mid s_{3}\right) P\left(s_{3}\right), \text { and then get } \\
& P\left(d \mid s_{1} S_{2} s_{3}\right)=P\left(s_{1} S_{2} S_{3} \mid d\right) P(d) / P\left(s_{1} S_{2} S_{3}\right)=
\end{aligned}
$$

$P\left(s_{1} S_{2} s_{3} \mid d\right) P(d) /\left(P\left(s_{1} \mid s_{2} S_{3}\right) P\left(s_{2} \mid s_{3}\right) P\left(s_{3}\right)\right)$.If all $\mathrm{s}_{\mathrm{k}}(\mathrm{k}=1, \ldots, 5)$ are independent, then we also get $P\left(d \mid s_{1} S_{2} S_{3}\right)=P\left(s_{1} S_{2} S_{3} \mid d\right) P(d) /\left(P\left(s_{1}\right) P\left(s_{2}\right) P\left(s_{3}\right)\right)$, and then get $P\left(d_{1} \mid s_{1} S_{2} S_{3} s_{4}\right)=P\left(s_{1} S_{2} S_{3} S_{4} \mid d_{1}\right) P\left(d_{1}\right) /\left(P\left(s_{1}\right) P\left(s_{2}\right) P\left(s_{3}\right) P\left(s_{4}\right)\right)$, and $P\left(d_{2} \mid s_{1} s_{2} s_{5}\right)=P\left(s_{1} s_{2} s_{5} \mid d_{2}\right) P\left(d_{2}\right) /\left(P\left(s_{1}\right) P\left(s_{2}\right) P\left(s_{5}\right)\right)$.

\section{Analysis and Discussion}

As to the computational complexity, as shown in Fig.1, before the improvement: $\mathrm{C}_{1}$ node calls child nodes (rules) for four times, $\mathrm{C}_{2}$ calls child nodes for three times, they share two rules (nodes). Upon improvement, the calling number of $\mathrm{C}_{1}$ is subject to no change, but $\mathrm{C}_{2}$ calls only one rule. In general, if node $C_{1}, \ldots, C_{k}, \ldots, C_{n}$ exist, and then each or more nodes in the $C_{k}(1<k<$ n) may share one or more child nodes, and $\mathrm{C}_{\mathrm{k}}(1<\mathrm{k}<\mathrm{n})$ can also have many parent nodes; so the computational complexity of the function depends on the number of nodes (rules) and calling relationship between rules. When the total of child nodes is large and relationship of rules is complex, the computational complexity is high, the improvement can reduce the computational complexity; in this sense, it should be significant.

When number of the analysed nodes is small, the need for storage space is less and the computational efficiency is almost not effected; but when the number is large, dozens of or even more, layered, classification and simplicity of designed rules should be considered. As to the transformer fault diagnosis, dozens of analyzed nodes and even more should be considered commonly, but sometimes, a dozen nodes can give the basic diagnosis, which depends on the accuracy of the rule knowledge. As to the transformer, multi-level nodes of bayesian network rule should be built. After some fault probabilities of components in a transformer are calculated, the fault probability of the transformer should be given. Table 1 corresponds to the part rules of the transformer overheating fault (or defect) cause analysis. The first column values in Table 2 is corresponding to the prior probabilities of the events described by the first column (via Column 5) in Table 1, and the prior probabilities are some experience values. The second column values in Table 2 are given according to site situation during system evaluation. The values of INC and DEC use for reference to literature [7].

\begin{tabular}{|l|l|l|l|l|}
\hline $\begin{array}{l}\text { Fault diagnosis rule description } \\
\text { (proposition) }\end{array}$ & $\begin{array}{l}\text { Belong to } \\
\text { conclusion }\end{array}$ & $\begin{array}{l}\text { Contain } \\
\text { condition }\end{array}$ & Node & Symbol \\
\hline $\begin{array}{l}\text { Chromatographic shows high temperature } \\
\text { overheating }\end{array}$ & $\mathrm{C}_{1}$ or $\mathrm{C}_{2}$ & & $\mathrm{X}_{1}$ & $S_{1}$ \\
\hline Total hydrocarbon is growing rapidly & $\mathrm{C}_{1}$ or $\mathrm{C}_{2}$ & & $\mathrm{X}$ & $S_{2}$ \\
\hline $\begin{array}{l}\text { DC resistance is not stable and larger } \\
\text { deviation }\end{array}$ & $\mathrm{C}_{1}$ & & $\mathrm{X}_{3}$ & $S_{3}$ \\
\hline $\begin{array}{l}\text { Total hydrocarbon continue to produce } \\
\text { under low voltage excitation }\end{array}$ & $\mathrm{C}_{1}$ & & $\mathrm{X}_{4}$ & $S 4$ \\
\hline $\begin{array}{l}1.1 \text { times overcurrent makes the oil } \\
\text { chromatogram obvious increased }\end{array}$ & $\mathrm{C}_{2}$ & $\mathrm{X}_{5}$ & $S_{5}$ \\
\hline Structure or magnetic shield short circuit & & $\begin{array}{l}\mathrm{X}_{1} \mathrm{X}_{2} \mathrm{X}_{3} \\
\mathrm{X}_{4}\end{array}$ & $\mathrm{C}_{1}$ & $d_{1}$ \\
\hline $\begin{array}{l}\text { Eddy current winding connect or weld poor } \\
\text { contact }\end{array}$ & & $\mathrm{X}_{1} \mathrm{X}_{2} \mathrm{X}_{5}$ & $\mathrm{C}_{2}$ & $d_{2}$ \\
\hline
\end{tabular}

Table 1: Part of Transformer Component Fault Diagnosis Rules Knowledge Base 


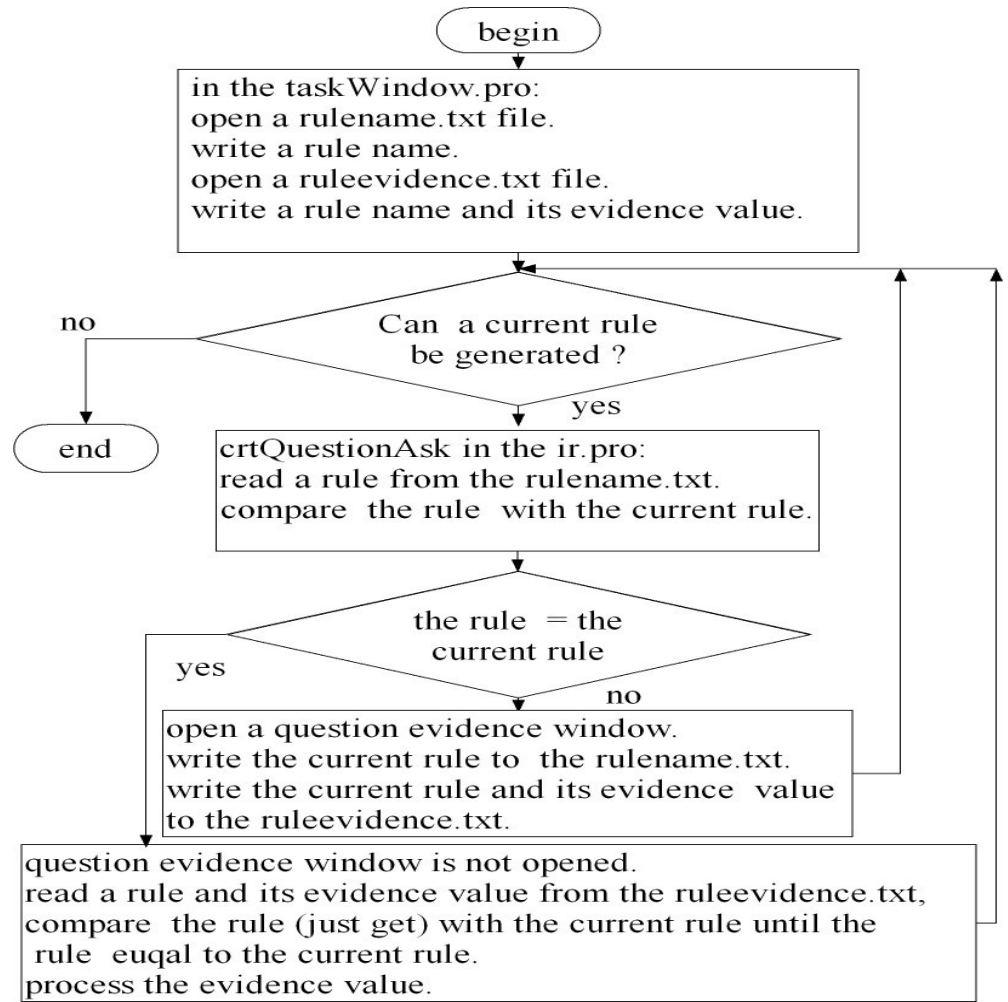

Figure 2: Evaluation Flowchart of Improved Bayesian Network Rule

\begin{tabular}{|c|l|l|c} 
& \multicolumn{1}{|c|}{ Argument } & \multicolumn{1}{c|}{ Data Element } & \multicolumn{1}{c}{ Value } \\
\hline 1 & diagnosis results & Structure or magnetic shield short circuit & 0.0068 \\
\hline 2 & diagnosis results & eddy current winding connect or weld poor contact & 0.0137 \\
\hline 3 & transformer & type & transformer \\
\hline
\end{tabular}

Figure 3: Result Example of Diagnostic Reasoning

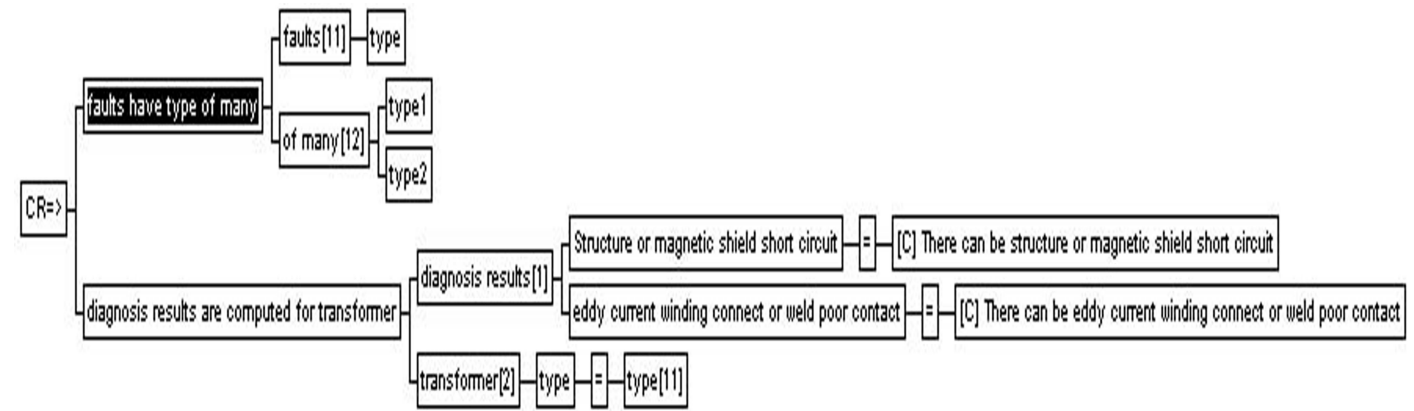

Figure 4: Sentence Rule of Calling to the Bayesian Network Rules 
There can be structure or magnetic shield short circuit

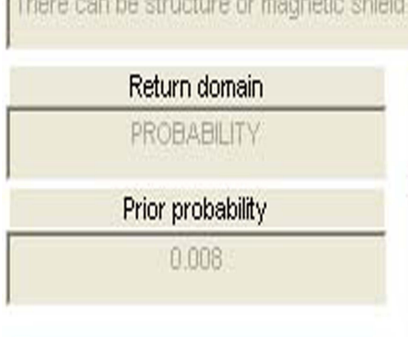

cTherCanBeStruOrMagnshieshorCirc

Rules in bavesian combination

Add Call

Remove Call

Edit Call

Move Up

Move Di

Chromatographic shows high temperature overheating [] :: INC: 1.90 DEC: 0.10

DC resistance is not stable and have larger deviation []:: INC: $1.50 \mathrm{DEC}: 0.50$

Total hydrocarbon can also continue to produce under low voltage excitation [].: INC: $1.30 \mathrm{DEC}: 0.70$

Total hydrocarbon is growing rapidly []:: INC: $1.70 \mathrm{DEC}: 0.30$

Figure 5: Definition Graph of Rule "Structure or Magnetic Shield Short Circuit"

\begin{tabular}{|c|c|c|c|}
\hline $\begin{array}{l}\text { Prior } \\
\text { probability(PP) }\end{array}$ & $\begin{array}{l}\text { Evaluated } \\
\text { probability }(\mathrm{P})\end{array}$ & $\begin{array}{l}S_{1}: \mathrm{INC}=1.1 \mathrm{DEC}=0.9 \\
s_{2}: \mathrm{INC}=1.3 \mathrm{DEC}=0.7 \\
s_{3}: \mathrm{INC}=1.5 \mathrm{DEC}=0.5 \\
s_{4}: \mathrm{INC}=1.7 \mathrm{DEC}=0.3 S_{5} \\
: \mathrm{INC}=1.5 \mathrm{DEC}=0.5\end{array}$ & $\begin{array}{c}S_{1}: \mathrm{INC}=1.9 \mathrm{DEC}=0.1 \quad S_{2} \\
: \mathrm{INC}=1.7 \mathrm{DEC}=0.3 \quad S_{3} \\
: \mathrm{INC}=1.5 \mathrm{DEC}=0.5 \quad S_{4} \\
: \mathrm{INC}=1.3 \mathrm{DEC}=0.7 \\
s_{5}: \mathrm{INC}=1.5 \mathrm{DEC}=0.5\end{array}$ \\
\hline$P\left(s_{1}\right)=0.035$ & $P\left(s_{1}\right)=0.035$ & \multirow{7}{*}{$\begin{array}{l}P\left(d_{1} \mid S_{1} S_{2} S_{3} S_{4}\right)=0.0075 \\
P\left(d_{2} \mid S_{1} S_{2} S_{5}\right)=0.0150\end{array}$} & \multirow{7}{*}{$\begin{array}{l}P\left(d_{1} \mid s_{1} S_{2} S_{3} S_{4}\right)=0.0068 \\
P\left(d_{2} \mid s_{1} S_{2} s_{5}\right)=0.0137\end{array}$} \\
\hline$P\left(s_{2}\right)=0.063$ & $P\left(s_{2}\right)=0.050$ & & \\
\hline$P\left(s_{3}\right)=0.045$ & $P\left(s_{3}\right)=0.046$ & & \\
\hline$P\left(s_{4}\right)=0.015$ & $P\left(s_{4}\right)=0.020$ & & \\
\hline$P\left(s_{5}\right)=0.023$ & $P(s 5)=0.025$ & & \\
\hline$P\left(d_{1}\right)=0.008$ & only for five & & \\
\hline$P\left(d_{2}\right)=0.016$ & que & & \\
\hline
\end{tabular}

Table 2: the $P\left(d_{1} \mid s_{1} S_{2} S_{3} S_{4}\right), P\left(d_{2} \mid s_{1} S_{2} S_{5}\right)$ computed by Pro/3 under some cases of combination

\section{Part Improved Script of Bayesian Network Rule Function in the ir.pro}

crtQuestionAsk(SolRef,Rule,ParVals,QDom,Q,C,Tree) :-...;

globalVars::get_i("server") $<>1, \ldots$,

file5x::openread(fileSelector::in,"d:||rulename.txt"), file5x::readdevice(fileSelector::in),

not(file5x::eof(fileSelector::in)),

file5x::readln(Record),

readCellsy(Record,Lnew),

file5x::closefile(fileSelector::in),

cuitest(Cui,Rule,Lnew),

Cui<>"1",

Dlg=inexQuestion::new(),

Dlg:show(W,Question,Rule,[],Dom,DefS),

appendcui(Lnew,[Rule],Lnew2),

file5x::openwrite(fileSelector::out,"d:\|rulename.txt"),

file5x::writedevice(fileSelector::out),

writeCellsy(Lnew2),

file5x::closefile(fileSelector::out),

RetPars=globalVars::popRetPars(),

RetPars=[AnsType,Ans],

file5x::openappend(fileSelector::stdout,"d: \ruleevidence.txt"),

file5x::writedevice(fileselector::stdout),

file5x::write(Rule,"),

file5x::write(Ans,''n'),

file5x::closefile(fileSelector::stdout),...,

crtQuestionValidate(SolRef,Rule,ParVals,QDom,Question,[],Ans,Tree,C),!;

globalVars::get_i("server") $<>1, \ldots$, 
file5x::openread(fileSelector::stdin,"d:\|ruleevidence.txt"),

file5x::readdevice(fileSelector::stdin),

readAns(Rule,Reccui),

Re1=Reccui,

file5x::closefile(fileSelector::stdin),

globalVars::pushRetPars(["2",Re1]),.., crtQuestionValidate(SolRef,Rule,ParVals,QDom,Question, [],Ans, Tree,C), !.

\section{Conclusion}

By analyzing the operating mechanism of the Bayesian network rule in the Pro/3,the application research work has improved the Bayesian network rule function. This improvement makes it possible, as to some same rules, to ask the same evidence such as an evaluated probability or a certainty factor only once, which can avoid repetitive inquiry, adding simplicity and improving Pro/3 system performance. Based on this improvement, this work has also created the transformer component fault diagnosis knowledge base model such that using the model has implemented an expert system prototype system. The basic application shows effectiveness of the improvement. It also shows that the diagnostic reasoning has some auxiliary analysis functions for determining the possibility of transformer components fault under given some prior probabilities and evaluated probabilities (according to some actual situations). The modeling approach created by the Pro/3 can be also applied to a diagnosis or an analysis of other equipment. The key to transformer fault diagnosis in the Pro/ 3 is to create a precise knowledge base including relevant prior probability, which will be further studied in the future. Exploring the combination application of an expert system and Bayesian network has certain practical significance for establishing some assistance decision support systems.

\section{References}

[1] Li Bian, ChenYuan Bian. Review on intelligence fault diagnosis in power networks[J].Power System Prote-ction and Control. 42(3),146-153( 2014) (In Chinese)

[2] Yan Nie. Research of transformer fault diagnosis method based on bayesian networks[D].Baoding: North China Electric Power University(2007) (In Chinese)

[3] Hyun Joon Cho,Jong Keun Park. An Expert System for Fault Section Diagnosis of Power System susing Fuzzy Relations[J].IEEE Transaction on powersystem.12(1),342-347(1997)

[4] YiQin Wan. Transformer fault diagnosis based on probabilistic neural network[D]. Nanchang: Nanchang University (2007) (In Chinese)

[5] HongYan Gao. Fault Diagnosis of Transformer Based on Radial Basis Probabilistic Neural Networks[J].Coal Mine Machinery. 28(10),198-200( 2007) (In Chinese)

[6] Jens Hintze Holm. Pro/3- A Production System-type Expert System Shell[C],Visual Prolog Application \&Language Conference,Prolog Development Center,Faro,Portugal,pp,90-102（2006）

[7] Jens Hintze Holm, Pro/3 expert system shell, Information on http://www.ligaya.net/p3

[8] Corporate standards of State Grid (Q/GDW 169-2008) « Guide for Condition Evaluation of Oilimmersed Power Transformers(Reactors) ». China Electric Power Press, Beijing( 2008)(In Chinese) 